\title{
Weighted norm estimates for the maximal operator of the Laguerre functions heat diffusion semigroup
}

\author{
by \\ R. Macías (Santa Fe), C. Segovia (Buenos Aires) \\ and J. L. Torrea (Madrid)
}

\begin{abstract}
We obtain weighted $L^{p}$ boundedness, with weights of the type $y^{\delta}, \delta>-1$, for the maximal operator of the heat semigroup associated to the Laguerre functions, $\left\{\mathcal{L}_{k}^{\alpha}\right\}_{k}$, when the parameter $\alpha$ is greater than -1 . It is proved that when $-1<\alpha<0$, the maximal operator is of strong type $(p, p)$ if $p>1$ and $2(1+\delta) /(2+\alpha)<p<2(1+\delta) /(-\alpha)$, and if $\alpha \geq 0$ it is of strong type for $1<p \leq \infty$ and $2(1+\delta) /(2+\alpha)<p$.

The behavior at the end points of the intervals where there is strong type is studied in detail and sharp results about the existence or not of strong, weak or restricted types are given.
\end{abstract}

1. Introduction. The Laguerre polynomials $L_{k}^{\alpha}(y)$ are given by

$$
e^{-y} y^{\alpha} L_{k}^{\alpha}(y)=\frac{1}{k !} \frac{d}{d y^{k}}\left(e^{-y} y^{k+\alpha}\right)
$$

where $y$ is positive. We assume that $\alpha>-1$. The Laguerre polynomials $\left\{L_{k}^{\alpha}(y)\right\}_{k=0}^{\infty}$ form an orthogonal system with respect to the measure $e^{-y} y^{\alpha} d y$. More precisely,

$$
\int_{0}^{\infty} L_{k}^{\alpha}(y) L_{j}^{\alpha}(y) e^{-y} y^{\alpha} d y=\frac{\Gamma(k+\alpha+1)}{\Gamma(k+1)} \delta_{k j} .
$$

The Laguerre functions $\mathcal{L}_{k}^{\alpha}(y)$ are defined by

$$
\mathcal{L}_{k}^{\alpha}(y)=\left(\frac{\Gamma(k+1)}{\Gamma(k+\alpha+1)}\right)^{1 / 2} e^{-y / 2} y^{\alpha / 2} L_{k}^{\alpha}(y) .
$$

2000 Mathematics Subject Classification: 42A45, 42B15, 42B20, 42B25, 42C10.

Key words and phrases: heat and Poisson semigroups, Laguerre functions.

Research partially supported by Dirección General de Investigación, Ministerio de Ciencia y Tecnología, BFM2002-04013-C02-02 and Proyecto IALE (UAM-Banco Santander Central-Hispano).

The first author was partially supported by Universidad Nacional del Litoral, Argentina.

The second author was partially supported by CONICET, Argentina. 
Standard references for Laguerre functions and polynomials are [1], [9] and [10].

We define the heat diffusion kernel $W^{\alpha}(t, y, z)$ for $\alpha>-1, t>0, y>0$, and $z>0$ as

$$
W^{\alpha}(t, y, z)=\sum_{n=0}^{\infty} e^{-t(n+(\alpha+1) / 2)} \mathcal{L}_{n}^{\alpha}(y) \mathcal{L}_{n}^{\alpha}(z),
$$

and the heat diffusion integral $W^{\alpha} f(t, y)$ as

$$
W^{\alpha} f(t, y)=\int_{0}^{\infty} W^{\alpha}(t, y, z) f(z) d z .
$$

The heat diffusion integral $W^{\alpha} f(t, y)$ satisfies the semigroup property

$$
W^{\alpha} f\left(t_{1}+t_{2}, y\right)=\int_{0}^{\infty} W^{\alpha}\left(t_{1}, y, z\right) W^{\alpha} f\left(t_{2}, z\right) d z .
$$

The maximal operator $W^{\alpha, *}$ associated to the heat diffusion integral $W^{\alpha} f(t, y)$ is given by

$$
W^{\alpha, *} f(y)=\sup _{t>0}\left|W^{\alpha} f(t, y)\right| .
$$

We define the fractional maximal function $M_{\theta} f(y)$ for $0 \leq \theta<1$ as

$$
M_{\theta} f(y)=\sup _{h>0} \frac{1}{(2 h)^{1-\theta}} \int_{|z| \leq h}|f(y-z)| d z .
$$

If $\theta=0, M_{0} f(y)$ is the Hardy-Littlewood centered maximal function. It is well known that if $y^{\delta}$ is a weight with $-1<\delta<p-1$, then $M_{0}$ is of strong type $(p, p)$ for $p>1$ and of weak type $(1,1)$ if $p=1$ for the measure $y^{\delta} d y$. We will also need the right-sided maximal function

$$
M^{+} f(y)=\sup _{h>0} \frac{1}{h} \int_{y}^{y+h}|f(z)| d z
$$

We denote by $A_{p}$ the class of all weights $\omega(y)$ such that $M_{0}$ is of strong type $(p, p)$ for $p>1$, and of weak type for $p=1$, for the measure $\omega(y) d y$, and by $A_{p}^{+}$the class of all weights $\omega(y)$ such that $M^{+}$is of strong type $(p, p)$ for $p>1$, and of weak type for $p=1$, for the measure $\omega(y) d y$. It is well known that $A_{1} \subset A_{p}$ and $A_{1}^{+} \subset A_{p}^{+}$for every $p>1$. For $M^{+}$we need to know that it is of weak type $(1,1)$ for the measure $y^{\delta} d y$ for any $\delta>-1$. This is true because for $\delta \geq 0$ the weight is a non-decreasing function, and for $-1<\delta \leq 0$, because $M^{+} f(y) \leq 2 M_{0} f(y)$. As references see $[4]-[6]$.

The purpose of this paper is to study the action of the maximal operator $W^{\alpha, *}$ just defined on the spaces $L^{p}\left((0, \infty), y^{\delta} d y\right)$ for $\delta>-1$. For 
$\alpha \geq 0$ and $\delta=0$ the results we give here were obtained by Stempak in [8], and for $-1<\alpha<0$ and $\delta=0$ by Macías, Segovia and Torrea in [3]. Even if not explicitly stated, estimates obtained in [8, Section 3] give $L^{p}$ weighted results, $1<p<\infty$, with the power weights $y^{\delta}$, where $-1<\delta<p-1$. However, Theorem 1 in the present paper shows that for $\alpha \geq 0$ the $\delta$-range can be enlarged to $-1<\delta<(1+\alpha / 2) p-1$. For the case when $\alpha \geq 0$ and $\delta>0$ we can majorize $W^{\alpha,{ }^{*}} f(y)$ by a constant times $W^{0, *} f(y)$ and thus we obtain the strong type $(p, p)$ of $W^{\alpha, *}$ whenever $p>1+\delta>0$. However, we can do better; in fact, in Theorem 1 we show that $W^{\alpha, *}$ is of strong type $(p, p)$ for the possibly greater interval $p>1$ and $p>2(1+\delta) /(\alpha+2)$.

2. Statement of the results. Let $N_{\alpha}$ denote the interval

$$
N_{\alpha}= \begin{cases}\left(\frac{2(1+\delta)}{2+\alpha}, \frac{2(1+\delta)}{-\alpha}\right) \cap(1, \infty) & \text { if }-1<\alpha<0, \\ \left(\frac{2(1+\delta)}{2+\alpha}, \infty\right] \cap(1, \infty] & \text { if } \alpha \geq 0 .\end{cases}
$$

We will assume that $N_{\alpha}$ is not empty. This implies that $1+\delta+\alpha / 2>0$; if not otherwise stated, we assume this throughout. With this notation, we have

Theorem 1. Let $-1<\alpha<\infty$ and $-1<\delta<\infty$. If $p \in N_{\alpha}$, then the maximal operator $W^{\alpha, *}$ is of strong type $(p, p)$ with respect to the measure $y^{\delta} d y$, that is,

$$
\int_{0}^{\infty} W^{\alpha, *} f(y)^{p} y^{\delta} d y \leq C_{\alpha, \delta, p} \int_{0}^{\infty}|f(y)|^{p} y^{\delta} d y
$$

with a constant $C_{\alpha, \delta, p}$ depending on $\alpha, p$ and $\delta$ only.

The following theorem gives the behavior of $W^{\alpha, *}$ at the end points of $N_{\alpha}$. We set $a_{\alpha}=\max (1,2(1+\delta) /(2+\alpha))$ and $b_{\alpha}=2(1+\delta) /(-\alpha)$ if $-1<\alpha<0$, and $b_{\alpha}=\infty$ if $\alpha \geq 0$.

TheOrem 2. Let $\delta>-1$. At the end points of $N_{\alpha}$, we have:

(a) If $-1<\alpha<0$, then the operator $W^{\alpha, *}$ is of weak type and not of strong type $\left(b_{\alpha}, b_{\alpha}\right)$ with respect to the measure $y^{\delta} d y$.

(b) If $\alpha \geq 0$, then $W^{\alpha, *}$ is of strong type $(\infty, \infty)$ with respect to $y^{\delta} d y$.

(c) If $\alpha>-1$ and $a_{\alpha}=2(1+\delta) /(2+\alpha)$, then $W^{\alpha, *}$ is of restricted weak type and not of weak type $\left(a_{\alpha}, a_{\alpha}\right)$ with respect to $y^{\delta} d y$.

(d) If $\alpha>-1$ and $a_{\alpha}=1$, then $W^{\alpha, *}$ is of weak type and not of strong type $(1,1)$ with respect to $y^{\delta} d y$. 
REMARK 1. If $-1<\alpha<0$ and $2(1+\delta) /(-\alpha)=1$, then the interval $N_{\alpha}$ is empty. However, since $2(1+\delta) /(2+\alpha)<2(1+\delta) /(-\alpha)=1$, by the proof of part (d) of Theorem 2, the operator $W^{\alpha, *}$ is of weak type and not of strong type $(1,1)$ with respect to the measure $y^{\delta} d y$.

Remark 2. The results obtained in Theorem 2 do not depend on Theorem 1, and can be used to give a proof of Theorem 1 by interpolation (see [7] and [2]).

3. Lemmas. Throughout this paper we shall assume that $f$ is a nonnegative function. The constants will not have the same value at each occurrence.

Definition 1 . Let $f$ be a locally integrable function on $(0, \infty)$. We define the maximal function $M^{R} f$ for $0<y<\infty$ by

$$
M^{R} f(y)=\sup _{J_{y} \subset(y / 4,3 y)} \frac{1}{\left|J_{y}\right|} \int_{J_{y}} f(z) d z
$$

where $J_{y}$ runs over all intervals containing $y$. Obviously, $M^{R} f(y) \leq$ $C M_{0} f(y)$.

Lemma 1. The maximal function $M^{R}$ is of weak type $(p, p), 1 \leq p \leq \infty$, with respect to the measure $y^{\delta} d y$ for any real $\delta$.

Proof. The case $p=\infty$ is obvious. Let us represent $(0, \infty)$ as the union of the intervals $\left\{\left(8^{k}, 8^{k+1}\right)\right\}_{k=-\infty}^{\infty}$. If $y \in\left\{y: \lambda<M^{R} f(y)\right\} \cap\left(8^{k}, 8^{k+1}\right]$, then there exists an interval $J_{y}$ such that $y \in J_{y} \subset(y / 4,3 y)$ and

$$
M^{R} f(y) \leq 2 \frac{1}{\left|J_{y}\right|} \int_{J_{y}} f(z) d z
$$

This interval $J_{y}$ is contained in $\left(8^{k-1}, 8^{k+2}\right)$. Then, by Hölder's inequality,

$$
\lambda^{p}<M^{R} f(y)^{p} \leq\left(2 \frac{1}{\left|J_{y}\right|} \int_{J_{y}} f(z) d z\right)^{p} \leq 2^{p} \frac{1}{\left|J_{y}\right|} \int_{J_{y}} f(z)^{p} d z .
$$

Given a compact subset $K$ of $\left\{y: \lambda<M^{R} f(y)\right\} \cap\left(8^{k}, 8^{k+1}\right]$, we can find a finite sequence $\left\{J_{y_{i}}\right\}$ that covers $K$ and is such that no point of $K$ belongs to more than three intervals of the sequence. Then

$$
\begin{aligned}
\int_{K} y^{\delta} d y & \leq \sum_{i} \int_{J_{y_{i}}} y^{\delta} d y \leq c_{\delta} 8^{k \delta} \sum_{i}\left|J_{y_{i}}\right| \leq c_{\delta} 2^{p} 8^{k \delta} \frac{1}{\lambda^{p}} \sum_{i} \int_{J_{y_{i}}} f(z)^{p} d z \\
& \leq 3 c_{\delta} 2^{p} 8^{k \delta} \frac{1}{\lambda^{p}} \int_{\left(8^{k-1,8^{k+2}}\right)} f(z)^{p} d z \leq c_{\delta, p} \frac{1}{\lambda^{p}} \int_{\left(8^{\left.k-1,8^{k+2}\right)}\right.} f(z)^{p} z^{\delta} d z .
\end{aligned}
$$


Thus,

Hence,

$$
\int_{\left\{y: \lambda<M^{R} f(y)\right\} \cap\left(8^{k}, 8^{k+1}\right]} y^{\delta} d y \leq c_{\delta, p} \frac{1}{\lambda^{p}} \int_{\left(8^{k-1}, 8^{k+2}\right)} f(z)^{p} z^{\delta} d z .
$$

$$
\int_{\left\{y: \lambda<M^{R} f(y)\right\}} y^{\delta} d y \leq c_{\delta, p} \frac{1}{\lambda^{p}} \int_{0}^{\infty} f(z)^{p} z^{\delta} d z
$$

and Lemma 1 is proved.

Lemma 2 ([3, Lemma 1$])$. Given $0 \leq \beta<1$, there exists a constant $C_{\beta}$ such that for every $y>0$,

$$
\begin{aligned}
y^{-\beta / 2} M_{\beta}\left(f(z) z^{-\beta / 2}\right)(y) \leq & C_{\beta}\left\{y^{\beta / 2} M_{0}\left(f(z) z^{-\beta / 2}\right)(y)\right. \\
& \left.+y^{-\beta / 2} M_{0}\left(f(z) z^{\beta / 2}\right)(y)+M_{0} f(y)\right\} .
\end{aligned}
$$

We shall introduce some notation. Let us consider the generating function for the Laguerre polynomials

$$
\begin{aligned}
\sum_{n=0}^{\infty} \frac{\Gamma(n+1)}{\Gamma(n+\alpha+1)} & L_{n}^{\alpha}(y) L_{n}^{\alpha}(z) r^{n} \\
& =\frac{1}{1-r} e^{-r(z+y) /(1-r)}(r y z)^{-\alpha / 2} I_{\alpha}\left(2 \frac{(r y z)^{1 / 2}}{1-r}\right),
\end{aligned}
$$

where $0 \leq r<1$ and $I_{\alpha}(y)=e^{-i \alpha \pi / 2} J_{\alpha}(i y)$ is the modified Bessel function (see $[1$, p. 189, (20)]. Let

$$
Q_{\alpha}(y, z, r)=\sum_{n=0}^{\infty} \frac{\Gamma(n+1)}{\Gamma(n+\alpha+1)} e^{-y / 2} y^{\alpha / 2} L_{n}^{\alpha}(y) e^{-z / 2} z^{\alpha / 2} L_{n}^{\alpha}(z) r^{n+(\alpha+1) / 2}
$$

then, by $(3.3), Q_{\alpha}(y, z, r)$ is equal to

$$
\sum_{n=0}^{\infty} \mathcal{L}_{n}^{\alpha}(y) \mathcal{L}_{n}^{\alpha}(z) r^{n+(\alpha+1) / 2}=\frac{r^{1 / 2}}{1-r} e^{-(z+y) / 2} e^{-r(z+y) /(1-r)} I_{\alpha}\left(2 \frac{(r y z)^{1 / 2}}{1-r}\right) .
$$

This shows that $Q_{\alpha}\left(y, z, e^{-t}\right)=W^{\alpha}(t, y, z)$. Let

$$
e^{-t}=\left(\frac{1-s}{1+s}\right)^{2}
$$

then $0<s \leq 1$ if and only if $0<t \leq \infty$. If we define

then we get the expression

$$
R_{\alpha}(y, z, s)=Q_{\alpha}\left(y, z,\left(\frac{1-s}{1+s}\right)^{2}\right),
$$

$$
\begin{aligned}
& R_{\alpha}(y, z, s) \\
& =\frac{1}{2} \frac{1-s^{2}}{2 s} e^{-\frac{1}{4}(s+1 / s)\left(y^{1 / 2}-z^{1 / 2}\right)^{2}} e^{-\frac{1}{2}(s+1 / s)(y z)^{1 / 2}} I_{\alpha}\left(\frac{1-s^{2}}{2 s}(y z)^{1 / 2}\right) .
\end{aligned}
$$


Observe also that

$$
\begin{aligned}
& W^{\alpha} f(t, y)=\int_{0}^{\infty} R_{\alpha}(y, z, s) f(z) d z \\
& \quad \text { for } s=\left(1-e^{-t / 2}\right) /\left(1+e^{-t / 2}\right)=\tanh (t / 4) .
\end{aligned}
$$

Moreover,

$$
1-s^{2}=4 e^{-t / 2} /\left(1+e^{-t / 2}\right)^{2} \leq 4 e^{-t / 2} .
$$

We shall need the following estimates for $I_{\alpha}(y)$ : for $\alpha>-1$, there exist two constants $c_{\alpha}$ and $C_{\alpha}$ such that (see $[1$, p. $5,(12)]$ and $[1$, p. 86, (5)])

$$
\text { (1) If } 0 \leq y \leq 1 \text {, then } c_{\alpha} y^{\alpha} \leq I_{\alpha}(y) \leq C_{\alpha} y^{\alpha} \text {. }
$$

(2) If $y \geq 1, \quad$ then $\quad c_{\alpha} \frac{1}{y^{1 / 2}} e^{y} \leq I_{\alpha}(y) \leq C_{\alpha} \frac{1}{y^{1 / 2}} e^{y}$.

Let

$$
D_{s}=\left\{y:\left(\frac{1-s^{2}}{2 s}\right)^{2} y \geq 1\right\} .
$$

By (3.7) and (3.4) we have

$$
\text { (3.8) } \quad \chi_{D_{s}}(y z) R_{\alpha}(y, z, s)
$$

$$
\begin{aligned}
& \leq C \frac{1}{2} \frac{1-s^{2}}{2 s} e^{-\frac{1}{4}(s+1 / s)\left(z^{1 / 2}-y^{1 / 2}\right)^{2}-\frac{1}{2}(s+1 / s)(z y)^{1 / 2}} \chi_{D_{s}}(y z) \frac{e^{\left(\frac{1-s^{2}}{2 s}\right)(z y)^{1 / 2}}}{\left(\frac{1-s^{2}}{2 s}\right)^{1 / 2}(z y)^{1 / 4}} \\
& \leq \frac{1}{2} \frac{1-s^{2}}{2 s} e^{-\frac{1}{4 s}\left(z^{1 / 2}-y^{1 / 2}\right)^{2}} \chi_{D_{s}}(y z) \frac{1}{\left(\frac{1-s^{2}}{2 s}\right)^{1 / 2}(z y)^{1 / 4}} .
\end{aligned}
$$

Here we have used the fact that

$$
-\frac{1}{2}\left(s+\frac{1}{s}\right)+\frac{1-s^{2}}{2 s}=-s .
$$

Analogously, by $(3.7), \chi_{D_{s}^{\mathrm{c}}}(y z) R_{\alpha}(y, z, s)$ is bounded by a constant times

$$
\frac{1}{2} \frac{1-s^{2}}{2 s} e^{-\frac{1}{4 s}\left(z^{1 / 2}-y^{1 / 2}\right)^{2}} \chi_{D_{s}^{\mathrm{c}}}(y z)\left(\frac{1-s^{2}}{2 s}(y z)^{1 / 2}\right)^{\alpha} .
$$

We define

$$
\begin{aligned}
& H_{\alpha, 1}(s, y)=\int_{0}^{\infty} \chi_{D_{s}}(y z) R_{\alpha}(y, z, s) f(z) d z \\
& H_{\alpha, 2}(s, y)=\int_{0}^{\infty} \chi_{D_{s}^{\mathrm{c}}}(y z) R_{\alpha}(y, z, s) f(z) d z .
\end{aligned}
$$

Given $y, s>0$, for every integer $k$ we define

$$
B_{k}(y)=\left\{z: 2^{k} s^{1 / 2}<\left|z^{1 / 2}-y^{1 / 2}\right| \leq 2^{k+1} s^{1 / 2}\right\}
$$


Let $k_{0}$ be an integer to be fixed later. Then

$$
\begin{aligned}
H_{\alpha, 1}(s, y) \leq & C_{\alpha} \sum_{k=-\infty}^{k_{0}} \frac{1-s^{2}}{2 s} e^{-2^{2 k} / 4} \int_{B_{k}(y)} \chi_{D_{s}}(y z) \frac{f(z) d z}{\left(\frac{1-s^{2}}{2 s}\right)^{1 / 2}(z y)^{1 / 4}} \\
& +C_{\alpha} \sum_{k=k_{0}+1}^{\infty} \frac{1-s^{2}}{2 s} e^{-2^{2 k} / 4} \int_{B_{k}(y)} \chi_{D_{s}}(y z) \frac{f(z) d z}{\left(\frac{1-s^{2}}{2 s}\right)^{1 / 2}(z y)^{1 / 4}} \\
= & H_{\alpha, 11}(s, y)+H_{\alpha, 12}(s, y) .
\end{aligned}
$$

For the same $k_{0}$ and $B_{k}(y), H_{\alpha, 2}(s, y)$ is bounded by a constant times

$$
\begin{aligned}
& \sum_{k=-\infty}^{k_{0}} \frac{1-s^{2}}{2 s} e^{-2^{2 k} / 4} \int_{B_{k}(y)} \chi_{D_{s}^{\mathrm{c}}}(y z)\left(\frac{1-s^{2}}{2 s}(y z)^{1 / 2}\right)^{\alpha} f(z) d z \\
& \quad+\sum_{k=k_{0}+1}^{\infty} \frac{1-s^{2}}{2 s} e^{-2^{2 k} / 4} \int_{B_{k}(y)} \chi_{D_{s}^{\mathrm{c}}}(y z)\left(\frac{1-s^{2}}{2 s}(y z)^{1 / 2}\right)^{\alpha} f(z) d z \\
& =H_{\alpha, 21}(s, y)+H_{\alpha, 22}(s, y) .
\end{aligned}
$$

Given $y, s>0$, let $k_{0}$ be the unique integer satisfying

$$
2^{k_{0}+2} s^{1 / 2}<y^{1 / 2} \leq 2^{k_{0}+3} s^{1 / 2} .
$$

If $k \leq k_{0}$ and $z \in B_{k}(y)$ then, since $\left|z^{1 / 2}-y^{1 / 2}\right| \leq 2^{k+1} s^{1 / 2}$, we get

$$
y / 4 \leq\left(y^{1 / 2}-2^{k+1} s^{1 / 2}\right)^{2} \leq z \leq\left(y^{1 / 2}+2^{k+1} s^{1 / 2}\right)^{2} \leq 3 y .
$$

In particular,

$$
y / 4 \leq z \leq 3 y .
$$

If $k \geq k_{0}$ and $z \in B_{k}(y)$, since $\left|z^{1 / 2}-y^{1 / 2}\right| \leq 2^{k+1} s^{1 / 2}$, we get

$$
0<z \leq 136 \cdot 2^{2 k} s \text { and } 0<y \leq 100 \cdot 2^{2 k} s .
$$

Lemma 3. Let $\alpha>-1$. We have the following estimates for the heat diffusion integral $W^{\alpha} f(t, y)$ :

(a) If $-1<\alpha \leq 0$, we set $\beta=-\alpha$. Then

$$
\begin{aligned}
& W^{\alpha} f(t, y) \\
& \quad \leq C_{\alpha}\left\{e^{-t / 4} M_{0} f(y)+e^{-t(1-\beta) / 2} y^{-\beta / 2} M_{\beta}\left(z^{-\beta / 2} f(z)\right)(y)\right\} .
\end{aligned}
$$

(b) If $\alpha \geq 0$, then

$$
\begin{aligned}
& W^{\alpha} f(t, y) \\
& \quad \leq C_{\alpha} e^{-t / 4}\left\{M^{R} f(y)+M^{+} f(y)+y^{-\alpha / 2} \frac{1}{y} \int_{0}^{y} z^{\alpha / 2} f(z) d z\right\} .
\end{aligned}
$$


Proof. We will estimate $H_{\alpha, 11}(s, y), H_{\alpha, 12}(s, y), H_{\alpha, 21}(s, y), H_{\alpha, 22}(s, y)$ for $\alpha>-1$. We observe that

$$
\sum_{k=-\infty}^{\infty} e^{-2^{2 k} / 4} 2^{\varrho k}<\infty \quad \text { if } \varrho>0 .
$$

Estimate of $H_{\alpha, 11}(s, y)$ for $\alpha>-1$. By (3.10), (3.13) and (3.12), $H_{\alpha, 11}(s, y)$ is less than or equal to a constant times the sum over $k \leq k_{0}$ of the terms

$$
\left(\frac{1-s^{2}}{2 s}\right)^{1 / 2} e^{-2^{2 k} / 4} y^{-1 / 2} \int_{\left(y^{1 / 2}-2^{k+1} s^{1 / 2}\right)^{2}}^{\left(y^{1 / 2}+2^{k+1} s^{1 / 2}\right)^{2}} f(z) d z .
$$

Clearly the above expression is bounded by a constant times

$$
\left(1-s^{2}\right)^{1 / 2} e^{-2^{2 k} / 4} 2^{k} \frac{1}{4 y^{1 / 2} 2^{k+1} s^{1 / 2}} \int_{\left(y^{1 / 2}-2^{k+1} s^{1 / 2}\right)^{2}}^{\left(y^{1 / 2}+2^{k+1} s^{1 / 2}\right)^{2}} f(z) d z .
$$

Then, considering $(3.12),(3.17)$ and $(3.1)$, and the fact $\left(y^{1 / 2}+2^{k+1} s^{1 / 2}\right)^{2}-$ $\left(y^{1 / 2}-2^{k+1} s^{1 / 2}\right)^{2}=4 y^{1 / 2} 2^{k+1} s^{1 / 2}$, we get

$$
H_{\alpha, 11}(s, y) \leq C_{\alpha}\left(1-s^{2}\right)^{1 / 2} M^{R} f(y) .
$$

Estimate of $H_{\alpha, 12}(s, y)$ for $\alpha>-1$. By $(3.10), H_{\alpha, 12}(s, y)$ is bounded by a constant times the sum over $k>k_{0}$ of the terms

$$
\frac{1-s^{2}}{2 s} e^{-2^{2 k} / 4} \int_{B_{k}(y)} \chi_{D_{s}}(y z) \frac{f(z) d z}{\left(\frac{1-s^{2}}{2 s}\right)^{1 / 2}(z y)^{1 / 4}} .
$$

The condition $\chi_{D_{s}}(y z)=1$ is equivalent to $z \geq \frac{1}{y}\left(\frac{2 s}{1-s^{2}}\right)^{2}$, and by (3.14), $y \leq 100 \cdot 2^{2 k} s$.

Let $\gamma \geq 0$. Then (3.19) is bounded by a constant times

$$
\begin{aligned}
\frac{1-s^{2}}{2 s} e^{-2^{2 k} / 4} & \int_{\frac{1}{y}\left(\frac{2 s}{1-s^{2}}\right)^{2}}^{100 \cdot 2^{2 k} s} \chi_{D_{s}}(y z) \frac{f(z) d z}{\left(\frac{1-s^{2}}{2 s}\right)^{1 / 2}(z y)^{1 / 4}} \\
= & \left(\frac{1-s^{2}}{2 s}\right) e^{-2^{2 k} / 4} \int_{\frac{1}{y}\left(\frac{2 s}{1-s^{2}}\right)^{2}}^{y} \chi_{D_{s}}(y z) \frac{z^{\gamma} f(z) d z}{\left(\frac{1-s^{2}}{2 s}\right)^{1 / 2}(z y)^{1 / 4} z^{\gamma}} \\
& +\left(\frac{1-s^{2}}{2 s}\right) e^{-2^{2 k} / 4} \int_{y}^{100 \cdot 2^{2 k} s} \chi_{D_{s}}(y z) \frac{f(z) d z}{\left(\frac{1-s^{2}}{2 s}\right)^{1 / 2}(z y)^{1 / 4}}
\end{aligned}
$$




$$
\begin{aligned}
\leq & C\left(1-s^{2}\right)^{1+2 \gamma} e^{-2^{2 k} / 4} 2^{2(1+2 \gamma) k} y^{-\gamma} \frac{1}{y} \int_{0}^{y} z^{\gamma} f(z) d z \\
& +C\left(1-s^{2}\right) e^{-2^{2 k} / 4} 2^{2 k} \frac{1}{1002^{2 k} s} \int_{y}^{100 \cdot 2^{2 k} s} f(z) d z .
\end{aligned}
$$

Thus, for any $\gamma \geq 0, H_{\alpha, 12}(s, y)$ is bounded by a constant times

$$
\left(1-s^{2}\right)^{1+2 \gamma} y^{-\gamma} \frac{1}{y} \int_{0}^{y} z^{\gamma} f(z) d z+\left(1-s^{2}\right) M^{+} f(y)
$$

This implies that for $\gamma=0$, we get

$$
H_{\alpha, 12}(s, y) \leq C_{\alpha}\left(1-s^{2}\right) M_{0} f(y)
$$

and for $\alpha \geq 0$, taking $\gamma=\alpha / 2$, we get

$$
\begin{aligned}
H_{\alpha, 12}(s, y) \leq & C_{\alpha}\left(1-s^{2}\right)^{1+\alpha} y^{-\alpha / 2} \frac{1}{y} \int_{0}^{y} z^{\alpha / 2} f(z) d z \\
& +\left(1-s^{2}\right) M^{+} f(y) .
\end{aligned}
$$

Estimate of $H_{\alpha, 21}(s, y)$ for $-1<\alpha<0$. Let $\beta=-\alpha$. By (3.11)-(3.13), $H_{\alpha, 21}(s, y)$ is bounded by a constant times the sum over $k \leq k_{0}$ of the terms

$$
\begin{aligned}
& \frac{1-s^{2}}{2 s} e^{-2^{2 k} / 4} \int_{B_{k}(y)} \chi_{D_{s}^{\mathrm{c}}}(y z)\left(\frac{1-s^{2}}{2 s}(y z)^{1 / 2}\right)^{\alpha} f(z) d z \\
& \leq C\left(\frac{1-s^{2}}{2 s}\right)^{1+\alpha} e^{-2^{2 k} / 4} y^{\alpha / 2} \int_{\left(y^{1 / 2}-2^{k+1} s^{1 / 2}\right)^{2}}^{\left(y^{1 / 2}+2^{k+1} s^{1 / 2}\right)^{2}} \chi_{D_{s}^{\mathrm{c}}}(y z) z^{\alpha / 2} f(z) d z .
\end{aligned}
$$

If $\frac{1-s^{2}}{2 s}(y y / 4)^{1 / 2}>1$, then for $z \geq y / 4$ we have $\frac{1-s^{2}}{2 s}(y z)^{1 / 2}>1$, thus $\chi_{D_{s}^{\mathrm{c}}}(y z)=0$ and the integral above is zero. Therefore, we can assume that $\frac{1-s^{2}}{2 s}(y y / 4)^{1 / 2} \leq 1$, which implies that

$$
\left(1-s^{2}\right) y \leq 4 s
$$

Therefore $H_{\alpha, 21}(s, y)$ is bounded by a constant times the sum over $k \leq k_{0}$ of the terms

$$
\begin{gathered}
\left(\frac{1-s^{2}}{2 s}\right)^{1+\alpha} e^{-2^{2 k} / 4} y^{\alpha / 2} \frac{\left(4 y^{1 / 2} 2^{k+1} s^{1 / 2}\right)^{1+\alpha}}{\left(4 y^{1 / 2} 2^{k+1} s^{1 / 2}\right)^{1+\alpha}} \int_{\left(y^{1 / 2}-2^{k+1} s^{1 / 2}\right)^{2}}^{\left(y^{1 / 2}+2^{k+1} s^{1 / 2}\right)^{2}} f(z) z^{\alpha / 2} d z \\
\leq C_{\alpha}\left(1-s^{2}\right)^{(1-\beta) / 2} e^{-2^{2 k} / 4} 2^{2 k(1+\alpha)} y^{-\beta / 2} M_{\beta}\left(f(z) z^{-\beta / 2}\right)(y)
\end{gathered}
$$

Thus, summing up over $k \leq k_{0}$, we get

$$
H_{\alpha, 21}(s, y) \leq C_{\alpha}\left(1-s^{2}\right)^{(1-\beta) / 2} y^{-\beta / 2} M_{\beta}\left(f(z) z^{-\beta / 2}\right)(y)
$$

for $-1<\alpha<0$. 
Estimate of $H_{\alpha, 21}(s, y)$ for $\alpha \geq 0$. By (3.11)-(3.13), $H_{\alpha, 21}(s, y)$ is bounded by a constant times the sum over $k \leq k_{0}$ of the terms

$$
\begin{aligned}
& \text { 25) } \frac{1-s^{2}}{2 s} e^{-2^{2 k} / 4} \int_{B_{k}(y)} \chi_{D_{s}^{\mathrm{c}}}(y z)\left(\frac{1-s^{2}}{2 s}(y z)^{1 / 2}\right)^{\alpha} f(z) d z \\
& \leq C_{\alpha}\left(\frac{1-s^{2}}{2 s}\right)^{1+\alpha} e^{-2^{2 k} / 4} y^{\alpha} \int_{\left(y^{1 / 2}-2^{k+1} s^{1 / 2}\right)^{2}}^{\left(y^{1 / 2}+2^{k+1} s^{1 / 2}\right)^{2}} \chi_{D_{s}^{\mathrm{c}}}(y z) f(z) d z \\
& =C_{\alpha}\left(\frac{1-s^{2}}{2 s}\right)^{1+\alpha} e^{-2^{2 k} / 4} y^{\alpha} \frac{4 y^{1 / 2} 2^{k+1} s^{1 / 2}}{4 y^{1 / 2} 2^{k+1} s^{1 / 2}} \int_{\left(y^{1 / 2}-2^{k+1} s^{1 / 2}\right)^{2}}^{\left(y^{1 / 2}+2^{k+1} s^{1 / 2}\right)^{2}} \chi_{D_{s}^{\mathrm{c}}}(y z) f(z) d z .
\end{aligned}
$$

By using (3.23) and the fact that $\alpha \geq 0$, we see that (3.25) is bounded by a constant times

$$
\begin{aligned}
&\left(1-s^{2}\right)^{1 / 2} \frac{e^{-2^{2 k} / 4} 2^{k}}{4 y^{1 / 2} 2^{k+1} s^{1 / 2}} \int_{\left(y^{1 / 2}-2^{k+1} s^{1 / 2}\right)^{2}}^{\left(y^{1 / 2}+2^{k+1} s^{1 / 2}\right)^{2}} f(z) d z \\
& \leq C\left(1-s^{2}\right)^{1 / 2} e^{-2^{2 k} / 4} 2^{k} M^{R} f(y) .
\end{aligned}
$$

Thus,

$$
H_{\alpha, 21}(s, y) \leq C_{\alpha}\left(1-s^{2}\right)^{1 / 2} M^{R} f(y) .
$$

Estimate of $H_{\alpha, 22}(s, y)$ for $-1<\alpha<0$. Let $\beta=-\alpha$. By (3.11) and (3.14), $H_{\alpha, 22}(s, y)$ is bounded by a constant times the sum over $k>k_{0}$ of the terms

$$
\begin{aligned}
& \frac{1-s^{2}}{2 s} e^{-2^{2 k} / 4} \int_{B_{k}(y)} \chi_{D_{s}^{\mathrm{c}}}(z y)\left(\frac{1-s^{2}}{2 s}(z y)^{1 / 2}\right)^{-\beta} f(z) d z \\
& \leq\left(\frac{1-s^{2}}{2 s}\right)^{1-\beta} e^{-2^{2 k} / 4} y^{-\beta / 2}\left(\frac{100 \cdot 2^{2 k} s}{100 \cdot 2^{2 k} s}\right)^{1-\beta} \int_{0}^{100 \cdot 2^{2 k} s} \chi_{D_{s}^{\mathrm{c}}}(z y) z^{-\beta / 2} f(z) d z .
\end{aligned}
$$

The above expression is smaller than or equal to a constant times

$$
\begin{array}{r}
\left(1-s^{2}\right)^{1-\beta} e^{-2^{2 k} / 4} 2^{2 k(1-\beta)} y^{-\beta / 2} \frac{1}{\left(100 \cdot 2^{2 k} s\right)^{1-\beta}} \int_{0}^{100 \cdot 2^{2 k} s} z^{-\beta / 2} f(z) d z \\
\leq\left(1-s^{2}\right)^{1-\beta} e^{-2^{2 k} / 4} 2^{2 k(1-\beta)} y^{-\beta / 2} M_{\beta}\left(z^{-\beta / 2} f(z)\right)(y) .
\end{array}
$$

Hence, for $-1<\alpha<0, H_{\alpha, 22}(s, y)$ is bounded by a constant times

$$
\left(1-s^{2}\right)^{1-\beta} y^{-\beta / 2} M_{\beta}\left(z^{-\beta / 2} f(z)\right)(y) .
$$


Estimate of $H_{\alpha, 22}(s, y)$ for $\alpha \geq 0$. By (3.11) and (3.14), $H_{\alpha, 22}(s, y)$ is bounded by a constant times the sum over $k>k_{0}$ of the terms

$$
\begin{aligned}
& \frac{1-s^{2}}{2 s} e^{-2^{2 k} / 4} \int_{B_{k}(y)} \chi_{D_{s}^{\mathrm{c}}}(y z)\left(\frac{1-s^{2}}{2 s}(y z)^{1 / 2}\right)^{\alpha} f(z) d z \\
& \leq\left(\frac{1-s^{2}}{2 s}\right)^{1+\alpha} e^{-2^{2 k} / 4} y^{\alpha / 2} \int_{0}^{y} \chi_{D_{s}^{\mathrm{c}}}(y z) z^{\alpha / 2} f(z) d z \\
& \quad+\left(\frac{1-s^{2}}{2 s}\right)^{1+\alpha} e^{-2^{2 k} / 4} y^{\alpha / 2} \int_{y}^{100 \cdot 2^{2 k} s} \chi_{D_{s}^{\mathrm{c}}}(y z) z^{\alpha / 2} f(z) d z \\
& \leq\left(\frac{1-s^{2}}{2 s}\right)^{1+\alpha} e^{-2^{2 k} / 4} y^{\alpha / 2} \int_{0}^{y} f(z) z^{\alpha / 2} d z \\
& \quad+\left(\frac{1-s^{2}}{2 s}\right)^{1+\alpha} e^{-2^{2 k} / 4} y^{\alpha / 2}\left(100 \cdot 2^{2 k} s\right)^{\alpha / 2} \int_{y}^{100 \cdot 2^{2 k} s} f(z) d z .
\end{aligned}
$$

Since $y \leq 100 \cdot 2^{2 k} s$, we find that $(3.28)$ is bounded by a constant times

$$
\begin{aligned}
& \left(1-s^{2}\right)^{1+\alpha} e^{-2^{2 k} / 4} 2^{2(1+\alpha) k} y^{-\alpha / 2} \frac{1}{y} \int_{0}^{y} z^{\alpha / 2} f(z) d z \\
& +\left(1-s^{2}\right)^{1+\alpha} e^{-2^{2 k} / 4} 2^{2(1+\alpha) k} \frac{1}{100 \cdot 2^{2 k} s} \int_{y}^{100 \cdot 2^{2 k} s} f(z) d z .
\end{aligned}
$$

Thus, we have shown that, for $\alpha \geq 0, H_{\alpha, 22}(s, y)$ is bounded by a constant times

$$
\left(1-s^{2}\right)^{1+\alpha}\left(y^{-\alpha / 2} \frac{1}{y} \int_{0}^{y} z^{\alpha / 2} f(z) d z+M^{+} f(y)\right) .
$$

Now, taking into account (3.5) and (3.6), part (a) of Lemma 3 follows from (3.18), (3.20), (3.24), (3.27), and part (b) follows from (3.18), (3.22), (3.26), and (3.29). Thus, Lemma 3 is proved.

\section{Proof of the main results}

Proof of Theorem 1. As usual, set $\beta=-\alpha$. By Lemma 3, we have

$$
W^{\alpha, *} f(y) \leq C_{\alpha}\left\{M_{0} f(y)+y^{-\beta / 2} M_{\beta}\left(z^{-\beta / 2} f(z)\right)(y)\right\} .
$$

Thus, applying Lemma 2 , we get

$$
W^{\alpha, *} f(y) \leq C_{\beta}\left\{y^{\beta / 2} M_{0}\left(f(z) z^{-\beta / 2}\right)(y)+y^{-\beta / 2} M_{0}\left(f(z) z^{\beta / 2}\right)(y)+M_{0} f(y)\right\} .
$$

The hypothesis "if $-1<\alpha<0$, then $p \in\left(a_{\alpha}, b_{\alpha}\right)$ " is equivalent to $-1<$ $\delta-p \beta / 2 \leq \delta+p \beta / 2<p-1$, and $p>1$. Under these conditions, the weights 
$y^{\delta+p \beta / 2}, y^{\delta-p \beta / 2}$ and $y^{\delta}$ belong to the class $A_{p}$ of Muckenhoupt, thus

$$
\begin{array}{r}
\int_{0}^{\infty}\left(y^{\beta / 2} M\left(f(z) z^{-\beta / 2}\right)(y)\right)^{p} y^{\delta} d y \leq C_{\alpha, p} \int_{0}^{\infty} f(y)^{p} y^{\delta} d y \\
\int_{0}^{\infty}\left(y^{-\beta / 2} M\left(f(z) z^{\beta / 2}\right)(y)\right)^{p} y^{\delta} d y \leq C_{\alpha, p} \int_{0}^{\infty} f(y)^{p} y^{\delta} d y \\
\int_{0}^{\infty} M_{0} f(y)^{p} y^{\delta} d y \leq C_{\alpha, p} \int_{0}^{\infty} f(y)^{p} y^{\delta} d y,
\end{array}
$$

proving that $W^{\alpha, *}$ is of strong type $(p, p)$ for $p \in N_{\alpha}$ with respect to the measure $y^{\delta} d y$ if $-1<\alpha<0$.

Now, let $\alpha \geq 0$. By (3.16) of Lemma 3, we have

$$
W^{\alpha} f(t, y) \leq C_{\alpha}\left\{M^{R} f(y)+M^{+} f(y)+y^{-\alpha / 2} \frac{1}{y} \int_{0}^{y} z^{\alpha / 2} f(z) d z\right\} .
$$

For $M^{R} f(y)$, by Lemma 1 , for any $p>1$ and any $\delta>-1$, we have

$$
\int_{0}^{\infty} M^{R} f(y)^{p} y^{\delta} d y \leq C_{p, \delta} \int_{0}^{\infty} f(y)^{p} y^{\delta} d y
$$

For $M^{+} f(y)$, since $y^{\delta} \in A_{1}^{+} \subset A_{p}^{+}$for any $\delta>-1$, as mentioned in the introduction, we have

$$
\int_{0}^{\infty} M^{+} f(y)^{p} y^{\delta} d y \leq C_{p, \delta} \int_{0}^{\infty} f(y)^{p} y^{\delta} d y
$$

Finally,

$$
y^{-\alpha / 2} \frac{1}{y} \int_{0}^{y} z^{\alpha / 2} f(z) d z \leq 2 y^{-\alpha / 2} M_{0}\left(f(z) z^{\alpha / 2}\right)(y) .
$$

Thus, if $-1<\delta-p \alpha / 2<p-1$ and $p>1$ we have

$$
\begin{aligned}
\int_{0}^{\infty}\left(y^{-\alpha / 2} \frac{1}{y} \int_{0}^{y} z^{\alpha / 2} f(z) d z\right)^{p} y^{\delta} d y & \leq \int_{0}^{\infty}\left(y^{-\alpha / 2} M_{0}\left(f(z) z^{\alpha / 2}\right)(y)\right)^{p} y^{\delta} d y \\
& \leq C_{p, \alpha, \delta} \int_{0}^{\infty} f(y)^{p} y^{\delta} d y .
\end{aligned}
$$

The conditions $-1<\delta-p \alpha / 2<p-1, p>1$ are equivalent to $p>$ $2(1+\delta) /(\alpha+2), p>1$, and $p<2(1+\delta) / \alpha$. In order to finish the proof we need to show that the condition $p<2(1+\delta) / \alpha$ can be removed.

Observe that $W^{0, *} f(y)$ is bounded by a constant times $M_{0} f(y)$, so $W^{0, *}$ is of strong type $(p, p)$ for $-1<\delta<p-1$ and $p>1$ with respect to the measure $y^{\delta} d y$. 
Assume that $0 \leq \alpha<2$ and $p \geq 2(1+\delta) / \alpha$. Then $p \geq 2(1+\delta) / \alpha>$ $2(1+\delta) /(0+2)=1+\delta$. Since by $(3.7)$ we have $W^{\alpha, *} f(y) \leq C_{\alpha} W^{0, *} f(y)$, it follows that $W^{\alpha, *}$ is of strong type $(p, p)$ for $p \geq 2(1+\delta) / \alpha$. We have showed the result for $\alpha$ in the range $0 \leq \alpha<0+2$. Now the result follows by induction on $[j, j+2)$.

Proof of Theorem 2. (a) If $-1<\alpha<0$ and $2(1+\delta) /(-\alpha)>1$, the upper end point of $N_{\alpha}$ is $2(1+\delta) /(-\alpha)$. For $s$ fixed, $0<s<1$, consider points $y$ and $z$ satisfying

$$
\frac{1-s^{2}}{2 s} y \leq 1 \text { and } \quad \frac{1-s^{2}}{2 s} z \leq 1
$$

By (3.4), using (3.7), we have

$$
R_{\alpha}(y, z, s) \geq C_{\alpha, s} y^{\alpha / 2} z^{\alpha / 2} .
$$

Thus, setting $a=2 s /\left(1-s^{2}\right)$, we get

$$
W^{\alpha}\left(\chi_{(0, a)}\right)(s, y) \geq C_{\alpha, s} y^{\alpha / 2} \int_{0}^{a} z^{\alpha / 2} d z=C_{\alpha, s} y^{\alpha / 2}
$$

for every $0 \leq y \leq a$. Since

$$
\int_{0}^{a}\left(y^{\alpha / 2}\right)^{2(1+\delta) /(-\alpha)} y^{\delta} d y=\int_{0}^{a} y^{-1} d y=\infty
$$

it follows that the operator $W^{\alpha, *}$ is not of strong type $(2(1+\delta) /(-\alpha)$, $2(1+\delta) /(-\alpha))$ with respect to the measure $y^{\delta} d y$. However, it is of weak type. In fact, let $\beta=-\alpha$; it will be enough to show that the three terms on the right hand side of (3.2) satisfy the weak type condition. Since $-1<\alpha<0$ implies $-1<\delta<2(1+\delta) /(-\alpha)-1$, the third term of (3.2) is of strong type $(2(1+\delta) /(-\alpha), 2(1+\delta) /(-\alpha))$ with respect to the measure $y^{\delta} d y$.

The first term is bounded by $y^{(-\alpha) / 2} M_{0}\left(f(z) z^{\alpha / 2}\right)(y)$ and since

$$
-1<((-\alpha) / 2) 2(1+\delta) /(-\alpha)+\delta<2(1+\delta) /(-\alpha)-1,
$$

the weight $y^{((-\alpha) / 2) 2(1+\delta) /(-\alpha)+\delta}$ is in $A_{2(1+\delta) /(-\alpha)}$. This shows that

$$
\int_{0}^{\infty}\left(y^{(-\alpha) / 2} M_{0}\left(f(z) z^{\alpha / 2}\right)(y)\right)^{2(1+\delta) /(-\alpha)} y^{\delta} d y \leq C_{\alpha, \delta} \int f(y)^{2(1+\delta) /(-\alpha)} y^{\delta} d y,
$$

which implies the strong type $(2(1+\delta) /(-\alpha), 2(1+\delta) /(-\alpha))$ of the first term of (3.2) with respect to the measure $y^{\delta} d y$.

Consider now the second term of (3.2). If we denote $2(1+\delta) /(-\alpha)$ by $p$, then $p^{\prime}=2(1+\delta) /(2(1+\delta)+\alpha)$. By Hölder's inequality, we obtain

$$
\begin{aligned}
& \frac{1}{(2 h)^{1+\alpha}} \int_{y}^{y+h} f(z) z^{\alpha / 2} d z \\
& \quad \leq \frac{1}{(2 h)^{1+\alpha}}\|f\|_{L^{p}\left((y, y+h), z^{\delta} d z\right)}\left\|z^{\alpha / 2-\delta}\right\|_{L^{p^{\prime}}\left((y, y+h), z^{\delta} d z\right)} .
\end{aligned}
$$


In order to estimate $\left\|z^{\alpha / 2-\delta}\right\|_{L^{p^{\prime}}\left((y, y+h), z^{\delta} d z\right)}$ we observe that

$$
\delta+(\alpha / 2-\delta) p^{\prime}>-1 \quad \text { and }\left(\delta+(\alpha / 2-\delta) p^{\prime}+1\right) / p^{\prime}=1+\alpha .
$$

Then $\left\|z^{\alpha / 2-\delta}\right\|_{L^{p^{\prime}}\left((y, y+h), z^{\delta} d z\right)} \leq c_{\delta, \beta}(y+h)^{1+\alpha}$. Thus, since $y \leq 2 h$, we have

$$
\begin{aligned}
\frac{1}{(2 h)^{1+\alpha}} \int_{y}^{y+h} f(z) z^{\alpha / 2} d z & \leq C_{\delta, \alpha}\left(\frac{y+h}{h}\right)^{1+\alpha}\|f\|_{L^{p}\left((y, y+h), z^{\delta} d z\right)} \\
& \leq C_{\delta, \alpha}\|f\|_{L^{p}\left((0, \infty), z^{\delta} d z\right)} .
\end{aligned}
$$

Multiplying by $y^{-\beta / 2}$ and taking the supremum over $h \geq y / 2$, we obtain

$$
\sup _{h \geq y / 2} y^{\alpha / 2} \frac{1}{(2 h)^{1+\alpha}} \int_{y}^{y+h} f(z) z^{-\beta / 2} d z \leq C_{\delta, \beta} y^{\alpha / 2}\|f\|_{L^{p}\left((0, \infty), z^{\delta} d z\right)} .
$$

From this inequality the weak type $(p, p)$ for $p=2(1+\delta) /(-\alpha)$ with respect to the measure $y^{\delta} d y$ is readily obtained.

(b) If $\alpha \geq 0$, the upper end point of $N_{\alpha}$ is $\infty$, and by (3.7) and (3.16), we have $W^{\alpha, *} f(y) \leq C_{\alpha} W^{0, *} f(y) \leq C_{\alpha} M_{0} f(y)$. Therefore since $L^{\infty}\left((0, \infty), y^{\delta} d y\right)$ $=L^{\infty}((0, \infty), d y)$ for $\delta>-1$, the operator $W^{\alpha, *}$ is of strong type $(\infty, \infty)$ with respect to the measure $y^{\delta} d y$.

(c) If the lower end point of $N_{\alpha}$ is greater than 1 , then it coincides with $2(1+\delta) /(2+\alpha)$. This implies that $2 \delta-\alpha>0$. If for a given $a>0$ the integral $\int_{0}^{a} f(z) z^{\alpha / 2} d z=\int_{0}^{a} f(z) z^{\alpha / 2-\delta} z^{\delta} d z$ is finite for every $f \in L^{2(1+\delta) /(2-\beta)}\left((0, a), z^{\delta} d z\right)$, then since

$$
\left(\frac{2(1+\delta)}{2+\alpha}\right)^{\prime}=\frac{2(1+\delta)}{2 \delta-\alpha}
$$

by uniform boundedness, it follows that $z^{\alpha / 2-\delta} \in L^{2(1+\delta) /(2 \delta-\alpha)}\left((0, a), z^{\delta} d z\right)$. This is a contradiction since $z^{(\alpha / 2-\delta) 2(1+\delta) /(2 \delta-\alpha)+\delta}=z^{-1}$. Therefore, there exists $f \in L^{2(1+\delta) /(2+\alpha)}\left((0, a), z^{\delta} d z\right)$ such that $\int_{0}^{a} f(z) z^{\alpha / 2} d z=\infty$. For this $f$, if $a=2 s /\left(1-s^{2}\right)$, then

$$
\int_{0}^{a} R(s, y, z) f(z) d z \geq C_{\alpha, s} y^{\alpha / 2} \int_{0}^{a} z^{\alpha / 2} f(z) d z=\infty,
$$

showing that $W^{\alpha, *} f(y)=\infty$ for every $y \leq a$. This tells us that the operator $W^{\alpha, *}$ cannot be of weak type at the lower end point $2(1+\delta) /(2-\beta)>1$ with respect to the measure $y^{\delta} d y$.

Now we shall prove the restricted type. Let $-1<\alpha<0$ and $\beta=-\alpha$. By (3.15) and Lemma 2, we have

$$
\begin{aligned}
& W^{\alpha, *} f(y) \\
& \quad \leq C_{\beta}\left\{M_{0} f(y)+y^{\beta / 2} \frac{1}{y} \int_{0}^{y} f(z) z^{-\beta / 2} d z+y^{-\beta / 2} M_{0}\left(f(z) z^{\beta / 2}\right)(y)\right\} .
\end{aligned}
$$


It is easy to see that

$$
\begin{aligned}
& -1<\delta<2(1+\delta) /(2-\beta)-1 \\
& -1<\delta-(\beta / 2) 2(1+\delta) /(2-\beta)<2(1+\delta) /(2-\beta)-1 .
\end{aligned}
$$

These inequalities imply that the weights $y^{\delta}$ and $y^{\delta-(\beta / 2) 2(1+\delta) /(2-\beta)}$ belong to $A_{2(1+\delta) /(2-\beta)}$. Therefore, the operators defined by

$$
M_{0} f(y) \text { and } y^{-\beta / 2} M_{0}\left(f(z) z^{\beta / 2}\right)(y)
$$

are of strong type $(2(1+\delta) /(2-\beta), 2(1+\delta) /(2-\beta))$ with respect to the measure $y^{\delta} d y$. We have not considered the second term of (4.1) yet. If $\alpha \geq 0$, by (3.16), we have

$$
W^{\alpha, *} f(y) \leq C_{\alpha}\left\{M^{R} f(y)+y^{-\alpha / 2} \frac{1}{y} \int_{0}^{y} z^{\alpha / 2} f(z) d z+M^{+} f(y)\right\}
$$

By Lemma 1, the first term on the right hand side of (4.2) is of weak type for any $p \geq 1$ and any $\delta>-1$. As mentioned in the introduction, the weight $y^{\delta}$ belongs to the class $A_{1}^{+} \subset A_{p}^{+}$of Sawyer for $\delta>-1$, so the operator $M^{+}$ is of weak type $(p, p)$ for any $p \geq 1$ with respect to the measure $y^{\delta} d y$ for any $\delta>-1$.

Now we are going to consider the second terms on the right hand side of both (4.1) and (4.2). They are of the form $y^{-\alpha / 2}(1 / y) \int_{0}^{y} z^{\alpha / 2} f(z) d z$ allowing $\alpha>-1$. Let $E$ be a measurable set contained in $(0, \infty)$ and $F$ the set defined by $\chi_{E}\left(u^{1 /(1+\delta)}\right)=\chi_{F}(u)$. By the change of variables $z=u^{1 /(1+\delta)}$, we have

$$
\int_{0}^{\infty} \chi_{E}(z) z^{\delta} d z=\frac{1}{1+\delta} \int_{0}^{\infty} \chi_{E}\left(u^{1 /(1+\delta)}\right) d u=\frac{1}{1+\delta}|F|,
$$

and

$$
\begin{aligned}
\int_{0}^{y} \chi_{E}(z) z^{\alpha / 2} d z & =\frac{1}{1+\delta} \int_{0}^{y^{1+\delta}} \chi_{E}\left(u^{1 /(1+\delta)}\right) u^{(\alpha / 2-\delta) /(1+\delta)} d u \\
& =\frac{1}{1+\delta} \int_{0}^{y^{1+\delta}} \chi_{F}(u) u^{(\alpha / 2-\delta) /(1+\delta)} d u
\end{aligned}
$$

Since $2(1+\delta) /(2+\alpha)>1$ implies $\alpha / 2-\delta<0$, it follows that

$$
\begin{aligned}
\int_{0}^{y^{1+\delta}} \chi_{F}(u) u^{(\alpha / 2-\delta) /(1+\delta)} d u & \leq \int_{0}^{\infty} \chi_{F}(u) u^{(\alpha / 2-\delta) /(1+\delta)} d u \\
& \leq \int_{0}^{|F|} u^{(\alpha / 2-\delta) /(1+\delta)} d u .
\end{aligned}
$$


Taking into account that $\alpha>-1$ implies $(\alpha / 2-\delta) /(1+\delta)>-1$, we can compute the last integral above, obtaining

$$
\int_{0}^{|F|} u^{(\alpha / 2-\delta) /(1+\delta)} d u=\frac{2(1+\delta)}{\alpha+2}|F|^{(\alpha+2) / 2(1+\delta)} .
$$

Then, by (4.3), we get

$$
\begin{aligned}
\int_{0}^{|F|} u^{\alpha / 2-\delta /(1+\delta)} d u & =\frac{2(1+\delta)}{2-\beta}\left((1+\delta) \int_{0}^{\infty} \chi_{E}(z) z^{\delta} d z\right)^{(\alpha+2) / 2(1+\delta)} \\
& =c_{\alpha, \delta}\left(\int_{0}^{\infty} \chi_{E}(z) z^{\delta} d z\right)^{(\alpha+2) / 2(1+\delta)}
\end{aligned}
$$

In consequence,

$$
y^{-\alpha / 2} \frac{1}{y} \int_{0}^{y} \chi_{E}(z) z^{\alpha / 2} d z \leq c_{\alpha, \delta} y^{-\alpha / 2} \frac{1}{y}\left(\int_{0}^{\infty} \chi_{E}(u) u^{\delta} d u\right)^{(\alpha+2) / 2(1+\delta)} .
$$

From this, the restricted weak type $(2(1+\delta) /(2+\alpha), 2(1+\delta) /(2+\alpha))$ for the operator $W^{\alpha, *} f$ with respect to the measure $y^{\delta} d y$ is readily obtained.

(d) Let us show that if the lower end point of $N_{\alpha}$ is 1 , then the operator $W^{\alpha, *}$ cannot be of strong type $(1,1)$ with respect to the measure $y^{\alpha} d y$. In fact, by (3.7), we have

$$
\begin{aligned}
& \chi_{D_{s}}(y z) R_{\alpha}(y, z, s) \\
& \quad \geq C_{\alpha}\left(\frac{1-s^{2}}{2 s}\right)^{1 / 2} e^{-\frac{1}{4 s}\left(y^{1 / 2}-z^{1 / 2}\right)^{2}} e^{-\frac{s}{4}\left(y^{1 / 2}-z^{1 / 2}\right)^{2}} e^{-s(y z)^{1 / 2}} \chi_{D_{s}}(y z) \frac{1}{(y z)^{1 / 4}} .
\end{aligned}
$$

Take $0<\varepsilon \leq 1$. Assume that $1<z \leq 1+\varepsilon, 1+2 \varepsilon \leq y \leq 2$, and $s=(y-1)^{2} / 4$. Then $s \leq 1 / 4, \frac{1-s^{2}}{2 s} \geq 1$, and $\left(\frac{1-s^{2}}{2 s}\right)^{1 / 2}(y z)^{1 / 4} \geq 1$. Thus $\chi_{D_{s}}(y z)=1$ and since

$$
\begin{aligned}
\frac{1}{4 s}\left(y^{1 / 2}-z^{1 / 2}\right)^{2} & =\frac{\left(y^{1 / 2}-z^{1 / 2}\right)^{2}}{(y-1)^{2}} \leq\left(\frac{(y-z)}{2(y-1)}\right)^{2} \\
& \leq\left(\frac{1}{2}+\frac{|1-z|}{2(y-1)}\right)^{2} \leq\left(\frac{1}{2}+\frac{\varepsilon}{4 \varepsilon}\right)^{2} \leq 1,
\end{aligned}
$$

we get $R_{\alpha}(y, z, s) \geq C_{\alpha} /(y-1)$, and therefore

$$
W^{\alpha, *}\left(\chi_{(1,1+\varepsilon)}\right)(y) \geq \frac{C_{\alpha}}{y-1} \int_{0}^{\infty} \chi_{(1,1+\varepsilon)}(z) d z=C_{\alpha} \frac{\varepsilon}{y-1}
$$

for $1+2 \varepsilon \leq y \leq 2$. Thus, if $W^{\alpha, *}$ were of strong type $(1,1)$ with respect to 
$y^{\delta} d y$, and recalling that $\delta>-1$, we would have

$$
\begin{aligned}
\int_{0}^{\infty} W^{\alpha, *}\left(\chi_{(1,1+\varepsilon)}\right)(y) y^{\delta} d y & \leq A_{\alpha} \int_{0}^{\infty} \chi_{(1,1+\varepsilon)}(y) y^{\delta} d y \\
& =A_{\alpha} \frac{(1+\varepsilon)^{1+\delta}-1}{1+\delta} \leq A_{\alpha, \delta} \varepsilon
\end{aligned}
$$

for a finite constant $A_{\alpha, \delta}$ depending on $\alpha$ and $\delta$ only. On the other hand, we get

$$
\begin{aligned}
& \int_{1+2 \varepsilon}^{2} W^{\alpha, *}\left(\chi_{(1,1+\varepsilon)}\right)(y) y^{\delta} d y \\
& \quad \geq C_{\alpha} \int_{1+2 \varepsilon}^{2} \frac{\varepsilon}{y-1} y^{\delta} d y \geq C_{\alpha, \delta} \int_{1+2 \varepsilon}^{2} \frac{\varepsilon}{y-1} d y=C_{\alpha, \delta} \varepsilon \log (1 / 2 \varepsilon) .
\end{aligned}
$$

In consequence, from (4.4) and (4.5), it follows that $C_{\alpha, \delta} \varepsilon \log (1 / 2 \varepsilon) \leq A_{\alpha} \varepsilon$, or also, that $C_{\alpha, \delta} \log (1 / 2 \varepsilon) \leq A_{\alpha, \delta}$. This is a contradiction since the left hand side of this inequality tends to $\infty$ as $\varepsilon$ tends to 0 , proving that $W^{\alpha, *}$ is not of strong type $(1,1)$ with respect to $y^{\delta} d y$.

However, as we are going to show, $W^{\alpha, *}$ is of weak type $(1,1)$ with respect to $y^{\delta} d y$. Since $2(1+\delta) /(2+\alpha) \leq 1$, it follows that $2 \delta-\alpha \leq 0$. Notice that since $N_{\alpha}$ is not empty we always have $2(1+\delta)+\alpha \geq 0$, which is equivalent to $1+\alpha+\delta-\alpha / 2 \geq 0$. Assume $-1<\alpha<0$, and let $\beta=-\alpha$. By (3.2) and (3.15) (Lemma 2), $W^{\alpha, *} f(y)$ is bounded by a constant times

$$
M_{0} f(y)+\sup _{y \leq 2 h}\left(\frac{y^{-\beta / 2}}{(2 h)^{1-\beta}} \int_{y}^{y+h} f(z) z^{-\beta / 2} d z\right)+y^{\beta / 2} \frac{1}{y} \int_{0}^{y} f(z) z^{-\beta / 2} d z .
$$

Since $2 \delta+\beta=2 \delta-\alpha \leq 0$ it follows that $-1<\delta<-\beta / 2<0$. Thus, $M_{0}$ is of weak type $(1,1)$ with respect to $y^{\delta} d y$. For the second term of $(4.6)$, since $y \leq 2 h$ and $2 \delta+\beta \leq 0$, we have

$$
\begin{aligned}
\frac{y^{-\beta / 2}}{(2 h)^{1-\beta}} \int_{y}^{y+h} f(z) z^{-\beta / 2} d z & \leq \frac{y^{-\beta / 2}}{(2 h)^{1-\beta}} \int_{0}^{3 h} f(t) z^{\delta} z^{-(\delta+\beta / 2)} d z \\
& \leq y^{-\beta / 2} \frac{(3 h)^{-(\delta+\beta / 2)}}{(2 h)^{1-\beta}} \int_{0}^{3 h} f(z) z^{\delta} d z \\
& =c_{\alpha, \delta} y^{-\beta / 2} \frac{1}{h^{1-\beta+(\delta+\beta / 2)}} \int_{0}^{3 h} f(z) z^{\delta} d z \\
& \leq c_{\beta, \delta} \frac{1}{y^{1+\delta}} \int_{0}^{\infty} f(z) z^{\delta} d z,
\end{aligned}
$$

which clearly implies the weak type $(1,1)$ of the second term. 
We still have to estimate the third term of (4.6). For $\alpha \geq 0$, from (3.16) we see that $W^{\alpha, *} f(y)$ is bounded by

$$
C_{\alpha}\left\{M^{R} f(y)+M^{+} f(y)+y^{-\alpha / 2} \frac{1}{y} \int_{0}^{y} z^{\alpha / 2} f(z) d z\right\} .
$$

By Lemma 1, the first term of (4.8) is of weak type for any $1 \leq p \leq \infty$ for the measure $y^{\delta} d y$ for any $\delta$. As mentioned before, $y^{\delta} \in A_{1}^{+}$for any $\delta>-1$, therefore $M^{+}$is of weak type $(1,1)$ with respect to the same measure $y^{\delta} d y$. For the third terms of (4.6) and (4.8), for $\alpha>-1$ we have

$$
y^{-\alpha / 2} \frac{1}{y} \int_{0}^{y} z^{\alpha / 2} f(z) d z=y^{-\alpha / 2} \frac{1}{y} \int_{0}^{y} z^{\alpha / 2-\delta} f(z) z^{\delta} d z .
$$

Since $\alpha / 2-\delta \geq 0$, this expression is bounded by

$$
y^{-\alpha / 2} \frac{y^{\alpha / 2-\delta}}{y} \int_{0}^{y} f(z) z^{\delta} d z \leq \frac{1}{y^{1+\delta}} \int_{0}^{\infty} f(z) z^{\delta} d z .
$$

This inequality and (4.8) imply the $(1,1)$ weak type of the operator $W^{\alpha, *}$ with respect to the measure $y^{\delta} d y$.

Acknowledgements. We thank the referee for his suggestions, which have contributed to clarify and improve the paper. We also thank B. Viviani for her enlightening comments.

\section{References}

[1] A. Erdélyi, W. Magnus, F. Oberhettinger and F. G. Tricomi, Higher Transcendental Functions, Volume II, McGraw-Hill, 1953.

[2] M. de Guzmán, Real Variable Methods in Fourier Analysis, North-Holland Math. Stud. 46, North-Holland, 1981.

[3] R. A. Macías, C. Segovia and J. L. Torrea, Heat diffusion maximal operators for Laguerre semigroups with negative parameters, J. Funct. Anal., to appear.

[4] B. Muckenhoupt, Weighted norm inequalities for the Hardy maximal function, Trans. Amer. Math. Soc. 165 (1972), 207-226.

[5] E. Sawyer, Weighted inequalities for the one-sided Hardy Litllewood maximal functions, ibid. 297 (1986), 53-61.

[6] E. M. Stein, Singular Integrals and Differentiability Properties of Functions, Princeton Math. Ser. 30, Princeton Univ. Press, 1970.

[7] E. M. Stein and G. Weiss, Fourier Analysis on Euclidean Spaces, Princeton Math. Ser. 32, Princeton Univ. Press, 1971.

[8] K. Stempak, Heat-diffusion and Poisson integrals for Laguerre expansions, Tohoku Math. J. 46 (1994), 83-104.

[9] G. Szegö, Orthogonal Polynomials, Amer. Math. Soc. Colloq. Publ. 23, Amer. Math. Soc., 1939. 
[10] S. Thangavelu, Lectures on Hermite and Laguerre Expansions, Math. Notes 24, Princeton Univ. Press, 1993.

IMAL-FIQ

CONICET-Universidad Nacional del Litoral

Güemes 3450

3000 Santa Fe, Argentina

E-mail: rmacias@ceride.gov.ar

Departamento de Matemáticas

Facultad de Ciencias

Universidad Autónoma de Madrid

28049 Madrid, Spain

E-mail: joseluis.torrea@uam.es
Instituto Argentino de Matemática

(IAM)-CONICET

Saavedra 15

1083 Buenos Aires, Argentina

E-mail: segovia@iamba.edu.ar

Received October 11, 2004

Revised version October 21, 2005 